\title{
Compact Accumulator using Lattices
}

\author{
Mahabir Prasad Jhanwar \\ R. C. Bose Center for Cryptology and Security \\ Indian Statistical Institute, India
}

\author{
Reihaneh Safavi-Naini \\ Department of Computer Science \\ University of Calgary, Canada
}

\begin{abstract}
An accumulator is a succinct aggregate of a set of values where it is possible to issue short membership proofs for each accumulated value. A party in possession of such a membership proof can then demonstrate that the value is included in the set. In this paper, we preset the first lattice-based accumulator scheme that issues compact membership proofs. The security of our scheme is based on the hardness of Short Integer Solution problem.
\end{abstract}

\section{Introduction}

Accumulators: An accumulator scheme $(\mathcal{A C})$ is a cryptographic authentication primitive for optimally verifying set-membership relations. Briefly, given a set $S$ of elements, an $\mathcal{A C}$ scheme can compute a short representation $\operatorname{Acc}(S)$ of $S$, called accumulation value, such that for every element $x \in S$ a short membership witness $w_{x}$ of " $x$ belonging to $S$ " can be generated. The accumulation value $\operatorname{Acc}(S)$ is published, and everybody can obtain it in an authenticated manner. Later, by exhibiting a valid $\left(x, w_{x}\right)$ pair, a prover can convince a verifier that the value $x$ was indeed accumulated into $\operatorname{Acc}(S)$. The security of the scheme requires that it be difficult to find a valid value-witness pair $\left(x^{*}, w_{x^{*}}\right)$ such that $x^{*} \notin S$. An accumulator is compact if it yields accumulation values and witnesses that are of constant size (i.e., independent of the number of elements $S$ contains).

Applications: Accumulators have proven to be a very strong mathematical tool with applications in a variety of privacy preserving technologies. Applications of accumulators include efficient time-stamping [BdM93], anonymous credential systems and group signatures [Nyb96, Ngu05, CKS09], ring signatures [DKNS04], redactable signatures [PS14], sanitizable signatures [CJ10], P-homomorphic signatures $\left[\mathrm{ABC}^{+} 12\right]$, and Zerocoin [MGGR] (an extension of the cryptographic currency Bitcoin), etc.

Evaluation: Accumulators were first introduced by Benaloh and de Mare [BdM93], and were later further studied and extended by Baric and Pfitzmann BP97. The security of both constructions was proved under the strong RSA assumption. Camenisch and Lysyanskaya CL02] augmented the latter work and proposed dynamic accumulators, in which elements can be efficiently added to and removed from the set of accumulated values, as well as privacy-preserving membership proofs. Alternative constructions of dynamic accumulators based on bilinear pairing Ngu05, DT08, CKS09], Paillier's trapdoor permutation [WWP08, and vector commitments CF13 are also known. Li et al. [LLX] introduced universal accumulators that extend the functionality of accumulators by supporting proofs that a given element is not a member of the set that has been accumulated. The security of their proposed instantiation is based on strong RSA assumption. Camacho et al. CHKO12 and Buldas et al. BLL00 independently introduced strong universal accumulators (also known as undeniable accumulators), which do not 
assume the accumulator manager is trusted. Both constructions were proved secure under the assumption that collision-resistant hash functions exists.

\subsection{Our Contribution}

In recent years, there has been rapid development in the use of lattices for constructing rich cryptographic schemes (these include digital signatures [GPV08, Boy10, CHKP12, identity-based encryption GPV08 and hierarchical IBE [CHKP12, ABB10], non-interactive zero knowledge [PV08], and even a fully homomorphic cryptosystem [Gen09]). Among other reasons, this is because such schemes have yet to be broken by quantum algorithms, and their security can be based solely on worst-case computational assumptions.

In the spirit of lattice-based cryptography, we present the first compact accumulator scheme from lattices and prove that it is secure based on the hardness of Short Integer Solution (SIS) problem. As the average-case SIS problem was shown to be as hard as certain worst-case lattice problems [Ajt96, MR07, GPV08], our scheme owns provable security under worst-case hardness assumption.

\subsection{Related Work}

Although, there exists no direct lattice-based $\mathcal{A C}$ scheme, the constructions in BdM93, BLL00, CHKO12 give indirect lattice-based instantiations because they only assume collision-resistant hash functions exist. This is true as lattice-based constructions of collision-resistant hash function are known [LM06, PR06], and therefore the security of the resulting schemes can also be reduced to worst-case assumptions on lattices. However, hash-tree based $\mathcal{A C}$ schemes are not compact as, in this setting, witnesses grows logarithmically with the number of elements in $S$.

\section{Preliminaries}

Notation: Let $\lambda \in \mathbb{N}$ be the security parameter and $1^{\lambda}$ its unary representation. We use standard asymptotic notation to describe the order of growth of functions. For any positive real valued functions $f(n)$ and $g(n)$ we write $f=O(g)$ if there exists two constants $c_{1}, c_{2}$ such that $f(n)<c_{1} \cdot g(n)$ for all $n \geq c_{2} ; f=\Omega(g)$ if $g=O(f) ; f=\Theta(g)$ if $f=O(g)$ and $g=O(f)$; and $f=o(g)$ if $\lim _{n \rightarrow \infty} \frac{f(n)}{g(n)}=0$. We denote $f=\tilde{O}(g)$ if $f=O(g \cdot \operatorname{poly}(\log g))$. The notation $\tilde{\Theta}$ is defined analogously. We denote $\omega(f(n))$ to denote a function that grows faster than $c \cdot f(n)$ for any $c>0$. We let poly $(n)$ denote an unspecified function $f(n)=O\left(n^{c}\right)$ for some constant $c$. A function $f(n)$ is called negligible, often written as $f(n)=\operatorname{negl}(n)$, if $f=o\left(\frac{1}{g}\right)$ for any polynomial $g=\operatorname{poly}(n)$. A function of $n$ is called overwhelming if it is $1-\operatorname{negl}(n)$. For a positive integer $k$, let $[k]$ denote the set $\{1, \ldots, k\}$. We denote the set of integers modulo $q$ by $\mathbb{Z}_{q}$, and identify it with the set $\{0, \ldots, q-1\}$ in the natural way. Column vectors are name by lower-case bold letters (e.g., $\boldsymbol{b}$ ) and matrices by upper-case bold letters (e.g., $\boldsymbol{B}$ ). For a matrix $\boldsymbol{S} \in \mathbb{R}^{m_{1} \times m_{2}}$, we call the norm of $\boldsymbol{S}$ as $\|\boldsymbol{S}\|=\max _{1 \leq i \leq m_{2}}|| \boldsymbol{s}_{i} \|$, where $\left\|\boldsymbol{s}_{i}\right\|$ denotes the $\ell_{2}$-norm (Euclidean norm) of the column vector $\boldsymbol{s}_{i}$. We let $\tilde{\boldsymbol{S}} \in \mathbb{R}^{m_{1} \times m_{2}}$ denotes the matrix whose columns $\tilde{\boldsymbol{s}}_{1}, \ldots, \tilde{\boldsymbol{s}}_{m_{2}}$ represents the Gram-Schmidt orthogonalization of the vectors $\boldsymbol{s}_{1}, \ldots, \boldsymbol{s}_{m_{2}}$ taken in the same order. Let $\|\tilde{\boldsymbol{S}}\|$ denote the Gram-Schmidt norm of $\boldsymbol{S}$. 


\section{$2.1 \quad$ Lattices}

Let $\mathbb{R}^{m}$ be the $m$-dimensional Euclidean space. A lattice $\Lambda \subseteq \mathbb{R}^{m}$ is a set

$$
\Lambda=\left\{\sum_{i=1}^{k} c_{i} \boldsymbol{b}_{i} \mid c_{i} \in \mathbb{Z} \text { and } \boldsymbol{b}_{1}, \ldots, \boldsymbol{b}_{k} \in \mathbb{R}^{m}\right\}
$$

of all integral combination of $k$ linearly independent vectors $\boldsymbol{b}_{1}, \ldots, \boldsymbol{b}_{k}$ in $\mathbb{R}^{m}(m \geq k) 1$. The integers $k$ and $m$ are called the rank and dimension of the lattice, respectively. The sequence of vectors $\boldsymbol{b}_{1}, \ldots, \boldsymbol{b}_{k}$ is called a lattice basis and it is conveniently represented as a matrix $\boldsymbol{B}=$ $\left[\boldsymbol{b}_{1}, \ldots, \boldsymbol{b}_{k}\right] \in \mathbb{R}^{m \times k}$ having the basis vectors as columns. Using the matrix notation, (1) can be written in a more compact form as $\Lambda=\Lambda(\boldsymbol{B})=\left\{\boldsymbol{B} \boldsymbol{c} \mid \boldsymbol{c} \in \mathbb{Z}^{k}\right\}$, where $\boldsymbol{B} \boldsymbol{c}$ is the usual matrix-vector multiplication. When $m=k$, the lattice is called full-rank. A lattice $\Lambda$ is called integer lattice if $\Lambda \subseteq \mathbb{Z}^{m}$. In this work, every lattice will be a full-rank lattice.

The minimum distance $\lambda_{1}(\Lambda)$ of a lattice $\Lambda$ is the length (Euclidean length, i.e., $\ell_{2}$ norm, unless otherwise indicated). More generally, the ith successive minimum $\lambda_{i}(\Lambda)$ is the smallest radius $r$ such that $\Lambda$ contains $i$ linearly independent vectors of norm at most $r$. The following are the two standard worst-case approximation problems on lattices: Shortest Vector Problem $\left(\mathrm{SVP}_{\gamma}\right)$ and Shortest Independent Vector Problem $\left(\mathrm{SIVP}_{\gamma}\right)$. In both problems, $\gamma=\gamma(m)$ is the approximation factor as a function of the lattice-dimension.

Definition $1\left(\mathrm{SVP}_{\gamma}\right)$ An input to $\mathrm{SVP}_{\gamma}$ is a basis $\boldsymbol{B}$ of a full-rank $m$-dimensional lattice. The goal is to output a nonzero lattice vector $\boldsymbol{B} \boldsymbol{x}$ (with $\boldsymbol{x} \in \mathbb{Z}^{m} \backslash\{\mathbf{0}\}$ ) such that $\|\boldsymbol{B} \boldsymbol{x}\| \leq \gamma \cdot\|\boldsymbol{B} \boldsymbol{y}\|$ for any $\boldsymbol{y} \in \mathbb{Z}^{m} \backslash\{\mathbf{0}\}$.

Definition $2\left(\mathrm{SIVP}_{\gamma}\right)$ An input to $\mathrm{SIVP}_{\gamma}$ is a basis $\boldsymbol{B}$ of a full-rank $m$-dimensional lattice. The goal is to output a set of $m$ linearly independent lattice vectors $\boldsymbol{B}_{\boldsymbol{x}_{1}}, \ldots, \boldsymbol{B} \boldsymbol{x}_{m} \in \Lambda(\boldsymbol{B})$ such that $\max _{i}\left\{\left\|\boldsymbol{B} \boldsymbol{x}_{i}\right\|\right\} \leq \gamma \cdot \lambda_{m}(\Lambda(\boldsymbol{B}))$.

\subsection{1 q-ary Lattices}

In this work we use $q$-ary lattices; a special family of full-rank integer lattices. A lattice from this family is most naturally specified not by a basis, but instead by a parity check matrix $\boldsymbol{A} \in \mathbb{Z}_{q}^{n \times m}$ for some positive integer $n$ and positive integer modulus $q$. The associated full rank lattice of dimensional $m$ is defined as:

$$
\Lambda^{\perp}(\boldsymbol{A})=\left\{\boldsymbol{x} \in \mathbb{Z}^{m} \mid \boldsymbol{A x}=\mathbf{0} \bmod q\right\}
$$

It is routine to check that $\Lambda^{\perp}(\boldsymbol{A})$ contains $\mathbf{0} \in \mathbb{Z}^{m}$ (thus non-empty) and is closed under subtraction, hence it is a lattice. The hardness of these lattices is most naturally parametrized by $n$ (not $m$, even though $m$ is the dimension of the lattices) and therefore it is standard to consider the parameters $m=m(n)$ and $q=q(n)$ as functions of $n$. By taking $m=c \cdot n \log q$ for some constant $c \geq 1$, it can be shown that with high probability, the minimum distance $\lambda_{1}\left(\Lambda^{\perp}(\boldsymbol{A})\right)$ of $\Lambda^{\perp}(\boldsymbol{A})$ is at most $\Theta(\sqrt{n \log q})$, where $\boldsymbol{A} \in \mathbb{Z}_{q}^{n \times m}$ is random.

Ajtai Ajt99, Alwen and Peikert AP09, Micciancio and Peikert MP12 provided methods to produce a matrix $\boldsymbol{A}$ statistically close to uniform in $\mathbb{Z}_{q}^{n \times m}$ along with a short basis $\boldsymbol{T}_{\boldsymbol{A}}$ of lattice $\Lambda^{\perp}(\boldsymbol{A})$. It is summarized in the following lemma.

\footnotetext{
${ }^{1}$ Alternatively, lattices can also be characterized without any reference to any basis. A lattice $\Lambda$ can be defined as a discrete nonempty subset of $\mathbb{R}^{m}$ which is closed under subtraction, i.e., if $\boldsymbol{x} \in \Lambda$ and $\boldsymbol{y} \in \Lambda$, then also $\boldsymbol{x}-\boldsymbol{y} \in \Lambda$. Here discrete means that there exists a positive real $\lambda>0$ such that the Euclidean distance between any two lattice vectors is at least $\lambda$.
} 
Proposition 1 (Short Basis Generation) There is a PPT algorithm that, on input a security parameter $1^{\lambda}$, an odd prime $q=\operatorname{poly}(\lambda)$, and two integers $n=\Theta(\lambda)$ and $m \geq 6 n \log q$, outputs a matrix $\boldsymbol{A} \in \mathbb{Z}_{q}^{n \times m}$ statistically close to uniform, and a basis $\boldsymbol{T}_{\boldsymbol{A}}$ for $\Lambda^{\perp}(\boldsymbol{A})$ with overwhelming probability such that $\left\|\tilde{\boldsymbol{T}}_{\boldsymbol{A}}\right\| \leq \tilde{\Theta}(\sqrt{m})$.

We refer to the algorithm of Proposition 1 by $\operatorname{TrapGen}\left(1^{\lambda}\right)$.

Primitive Matrix: We say that a matrix $\boldsymbol{A} \in \mathbb{Z}_{q}^{n \times m}$ is primitive if its columns generate all of $\mathbb{Z}_{q}^{n}$, i.e., $\boldsymbol{A} \cdot \mathbb{Z}^{m}(\bmod q)=\mathbb{Z}_{q}^{n}$. It is known that for any fixed constant $C>1$ and any $m \geq C n \log q$, a uniformly random $\boldsymbol{A} \in \mathbb{Z}_{q}^{n \times m}$ is primitive, except with $2^{-\Omega}(n)=\operatorname{negl}(n)$ probability. Therefore, throughout the paper we implicitly assume that such a uniform $\boldsymbol{A}$ is primitive.

\subsubsection{Hardness Assumption}

The short integer solution (SIS) problem was first suggested to be hard on average by Ajtai Ajt96 and later in [MR07] was formalized as follows. The security of our accumulator scheme is based on the hardness of this problem.

Definition 3 (SIS Problem) The small integer solution problem SIS (in the $\ell_{2}$ norm) is as follows: given an integer $q$, a matrix $\boldsymbol{A} \in \mathbb{Z}_{q}^{n \times m}$, and a real $\beta$, find an integer vector $\boldsymbol{e} \in \mathbb{Z}^{m}$ such that $\boldsymbol{A} \boldsymbol{e}=\mathbf{0} \bmod q$ and $\|\boldsymbol{e}\| \leq \beta$.

Clearly, the problem is syntactically equivalent to finding some short nonzero vector in $\Lambda^{\perp}(\boldsymbol{A})$. For functions $q(n), m(n)$, and $\beta(n)$, an average-case SIS problem instance is drawn from the probability ensemble over instances $(q(n), \boldsymbol{A}, \beta(n))$ where $\boldsymbol{A} \in \mathbb{Z}_{q}^{n \times m}$ is uniformly random. This average-case problem was shown to be as hard as certain worst-case lattice problems, first by Ajtai Ajt96], then by Micciancio and Regev [MR07], and Gentry et al. GPV08.

Theorem 1 ([GPV08]) For any poly-bounded $m$, any $\beta=\operatorname{poly}(n)$ and for any prime $q \geq$ $\beta \cdot \omega(\sqrt{n \log n})$, the average-case $\mathrm{SIS}_{q, m, \beta}$ is as hard as approximating the Shortest Independent Vector Problem $\left(\mathrm{SIVP}_{\gamma}\right)$, among others, in the worst-case to within certain $\gamma=\beta \cdot \tilde{O}(\sqrt{n})$ factors.

\subsubsection{Discrete Gaussian Distribution over Lattices}

For any $s>0$ the Gaussian function $\rho_{s, c}: \mathbb{R}^{m} \rightarrow \mathbb{R}$ centered at $\boldsymbol{c} \in \mathbb{R}^{m}$ with parameter $s$ is defined as:

$$
\forall \boldsymbol{x} \in \mathbb{R}^{m}, \rho_{s, \boldsymbol{c}}(\boldsymbol{x})=e^{-\frac{\pi\|\boldsymbol{x}-\boldsymbol{c}\|^{2}}{s^{2}}} .
$$

For any $c \in \mathbb{R}^{m}$, real $s>0$, and $m$-dimensional lattice $\Lambda$, define the discrete Gaussian distribution $D_{\Lambda, s, c}$ over $\Lambda$ (with center $\boldsymbol{c}$ and Gaussian parameter $s$ ) as:

$$
\forall \boldsymbol{x} \in \mathbb{R}^{m}, D_{\Lambda, s, \boldsymbol{c}}(\boldsymbol{x})=\frac{\rho_{s, \boldsymbol{c}}(\boldsymbol{x})}{\rho_{s, \boldsymbol{c}}(\boldsymbol{\Lambda})} .
$$

Micciancio and Regev MR07 proved that the norm $\left(\ell_{2}\right.$ norm) of vectors sampled from the discrete Gaussian distribution is small with high probability. We preset this result specialized to $q$-ary lattices.

Lemma 1 Let $\boldsymbol{A} \in \mathbb{Z}_{q}^{n \times m}$ be a primitive matrix, and $s$ be a Gaussian parameter with $s \geq$ $\omega(\sqrt{\log m})$. Then for $m$-dimensional full-rank lattice $\Lambda^{\perp}(\boldsymbol{A})$, and $\boldsymbol{c} \in \mathbb{R}^{m}$,

$$
\operatorname{Pr}_{\boldsymbol{x} \leftarrow D_{\Lambda}^{\perp}(\boldsymbol{A}), s, \boldsymbol{c}}[\|\boldsymbol{x}-\boldsymbol{c}\|>s \sqrt{m}] \leq \operatorname{negl}(m) .
$$

Gentry et al. GPV08 proved that, given a basis $\boldsymbol{B}$ for a lattice $\Lambda$, one can efficiently sample points in $\Lambda$ with discrete Gaussian distribution for sufficiently large values of $s$. 
Theorem 2 There is a PPT algorithm that, given a basis $\boldsymbol{B}$ of an $m$-dimensional lattice $\Lambda$, a parameter $s \geq\|\tilde{\boldsymbol{B}}\| \cdot \omega(\sqrt{\log m})$, and a center $\boldsymbol{c} \in \mathbb{R}^{m}$, outputs a sample from a distribution that is statistically close to $D_{\Lambda, s, c}$.

We refer to the algorithm of Theorem 2 by $\operatorname{SampleD}(\boldsymbol{B}, s, \boldsymbol{c})$.

The Gaussian Sampling Algorithm: SampleD $(\boldsymbol{B}, \boldsymbol{s}, \boldsymbol{c})$

- Input :

- a basis $\boldsymbol{B}$ of a lattice $\Lambda \subseteq \mathbb{R}^{m}$,

- a positive real parameter $s \geq\|\tilde{\boldsymbol{B}}\| \cdot \omega(\sqrt{\log m})$, and

- a center vector $c \in \mathbb{R}^{n}$.

- Output :

- a fresh random lattice vector $\boldsymbol{x} \in \Lambda$ drawn from a distribution statistically close to $D_{\Lambda, s, c}$.

We now recall an important lemma from [GPV08 which says that for a vector $\boldsymbol{e}$, chosen from an appropriate discrete Gaussian distribution over $\mathbb{Z}^{m}$, the vector $\boldsymbol{A e} \bmod q$ corresponds to a nearly-uniform element in $\mathbb{Z}_{q}^{n}$.

Lemma 2 Let $\boldsymbol{A} \in \mathbb{Z}_{q}^{n \times m}$ is primitive. Then for any $s \geq \omega(\sqrt{\log m})$, the distribution of $\boldsymbol{u}=$ Ae $\bmod q \in \mathbb{Z}_{q}^{n}$ is statistically close to uniform over $\mathbb{Z}_{q}^{n}$, where $\boldsymbol{e}$ is chosen from $D_{\mathbb{Z}^{m}, s, \mathbf{0}}$.

\subsubsection{Basis Delegation}

In CHKP12 a deterministic polynomial-time algorithm is given to extend a basis of $\Lambda^{\perp}(\boldsymbol{A})$ to a basis (without any loss of quality) of an arbitrary higher-dimensional extension $\Lambda^{\perp}(\boldsymbol{A} \| \overline{\boldsymbol{A}})$. We refer to this algorithm by BasisDel.

The Basis Delegation Algorithm: $\operatorname{Basis} \operatorname{Del}\left(\boldsymbol{T}_{\boldsymbol{A}}, \boldsymbol{A}, \overline{\boldsymbol{A}}\right)$

- Input :

- an arbitrary $\boldsymbol{A} \in \mathbb{Z}_{q}^{n \times m}$ such that $\boldsymbol{A}$ is primitive,

- an arbitrary basis $\boldsymbol{T}_{\boldsymbol{A}}$ of $\Lambda^{\perp}(\boldsymbol{A})$, and

- an arbitrary $\overline{\boldsymbol{A}} \in \mathbb{Z}_{q}^{n \times \bar{m}}$.

- Output :

- a basis $\boldsymbol{T}_{\boldsymbol{A}^{\prime}}$ of $\Lambda^{\perp}\left(\boldsymbol{A}^{\prime}=\boldsymbol{A} \| \overline{\boldsymbol{A}}\right) \subseteq \mathbb{Z}^{m+\bar{m}}$ such that $\left\|\tilde{\boldsymbol{T}}_{\boldsymbol{A}^{\prime}}\right\|=\left\|\tilde{\boldsymbol{T}}_{\boldsymbol{A}}\right\|$.

\subsubsection{Cryptographic Accumulators}

We now give a formal definition of a cryptographic accumulator scheme.

Definition 4 (Accumulator Scheme) Let $\mathcal{M}, \mathcal{C}$ and $\mathcal{W}$ be three sets (the message set, the set containing accumulated values and the set containing witnesses respectively). An accumulator scheme $\mathcal{A C}$ is a tuple of PPT algorithms (Setup, Accumulate, WitGen, Verify) with the following functionalities: 
- Setup $\left(1^{\lambda}\right)$ : Given a security parameter $\lambda$, it outputs a public key pk and a secret key sk. The remaining algorithms take pk as an implicit input.

- Accumulate $(X):$ If $X \subseteq \mathcal{M}$ then it accumulates all the elements of $X$ into an accumulation value $\operatorname{Acc}_{X} \in \mathcal{C}$.

- WitGen $(X, x, \mathrm{sk}):$ If $x \in X$ and $X \subseteq \mathcal{M}$, then it outputs a membership witness $w_{x} \in \mathcal{W}$; otherwise it outputs " $\perp$ " denoting Error.

- Verify $\left(x, w_{x}, c\right):$ For $x \in \mathcal{M}, w_{x} \in \mathcal{W}$ and $c \in \mathcal{C}$ it outputs either "1" denoting member or "0" denoting Error.

The correctness of an accumulator scheme requires that correctly accumulated values have valid witnesses with overwhelming probability, i.e., for $x \in \mathcal{M}, X \subseteq \mathcal{M}$, the verification algorithm $\operatorname{Verify}(x, \operatorname{Wit} \operatorname{Gen}(X, x$, sk), Accumulate $(X))$ outputs 1 with overwhelming probability if, $x \in X$.

Definition 5 (One-way Security) An accumulator scheme is one-way secure ${ }^{2}$ if, for all polynomial time adversaries $\mathcal{A}$ :

$$
\begin{gathered}
\operatorname{Pr}\left[\mathrm{pk} \leftarrow \operatorname{Setup}\left(1^{\lambda}\right) ;\left(X^{*}, x^{*}, w_{x^{*}}\right) \leftarrow \mathcal{A}(\mathrm{pk}) \mid x^{*} \notin X^{*} \subseteq \mathcal{M}\right. \text { and } \\
\left.\operatorname{Verify}\left(x^{*}, w_{x^{*}}, c \leftarrow \operatorname{Accumulate}\left(X^{*}\right)\right)=1\right] \leq \operatorname{negl}(\lambda) .
\end{gathered}
$$

If an accumulator satisfies this definition, then it is infeasible for an adversary to prove that a value $x$ was accumulated in a accumulation value $c$ when in fact it was not.

\section{A Compact Accumulator Scheme}

In this section we provide our accumulator scheme from lattices. Next, we discuss the correctness of our scheme. The security analysis of our scheme will be given in $\S 3.2$.

The parameters of our scheme involves:

- a security parameter $1^{\lambda}$;

- integers $n$ and $q$ (a prime) with $n=\Theta(\lambda)$ and $q=\operatorname{poly}(n)$;

- a dimension $m \geq 6 n \lg q$ and a bound $L=O(\sqrt{m})$;

- a Gaussian parameter $s \geq L \cdot \omega\left(\sqrt{\log \left(m+m^{\prime}\right)}\right)$, where $m^{\prime}=\operatorname{poly}(\lambda) \in \mathbb{N}$;

- a message set $\mathcal{M}=\left\{\boldsymbol{B}_{1}, \ldots, \boldsymbol{B}_{Q} \in \mathbb{Z}_{q}^{n \times m^{\prime}}\right\}$, where $Q=$ poly $(\lambda)$ and $\boldsymbol{B}_{i}$ 's are independently chosen with uniform distribution.

The scheme is defined as follows.

- Setup $\left(1^{\lambda}\right)$ : It uses the algorithm $\operatorname{TrapGen}\left(1^{\lambda}\right)$ from Proposition 1 to generate $\left(\boldsymbol{A}, \boldsymbol{T}_{\boldsymbol{A}}\right)$, where $\boldsymbol{A} \in \mathbb{Z}_{q}^{n \times m}$ is statistically close to uniform and $\boldsymbol{T}_{\boldsymbol{A}}$ is a short basis of $\Lambda^{\perp}(\boldsymbol{A})$ with $\left\|\tilde{\boldsymbol{T}}_{\boldsymbol{A}}\right\| \leq L$. The public key pk is set to $\boldsymbol{A}$, and the secret key sk is set to $\boldsymbol{T}_{\boldsymbol{A}}$. In the following, the other algorithms take pk $=\boldsymbol{A}$ as an implicit input.

- Accumulate $(X \subset \mathcal{M})$ : Without loss of generality, suppose $X=\left\{\boldsymbol{B}_{1}, \ldots, \boldsymbol{B}_{Q^{\prime}}\right\}$ for some $Q^{\prime} \in[Q]$. It accumulates the $Q^{\prime}$ matrices in the set $X$ into an compact accumulator value

$$
\operatorname{Acc}_{X}=\left[\sum_{\boldsymbol{B}_{i} \in X} \boldsymbol{B}_{i}\right] \in \mathbb{Z}_{q}^{n \times m^{\prime}} .
$$

\footnotetext{
${ }^{2}$ In the literature, the one-way secure accumulators are also known as collision-resistant accumulators.
} 
- WitGen $\left(X, \boldsymbol{B}\right.$, sk): Let $X=\left\{\boldsymbol{B}_{1}, \ldots, \boldsymbol{B}_{Q^{\prime}}\right\}$ for some $Q^{\prime} \in[Q]$. If $\boldsymbol{B} \notin X$, return $\perp$. Otherwise, $\boldsymbol{B} \in X$ and let $\boldsymbol{B}=\boldsymbol{B}_{j}$ for some $j \in\left[Q^{\prime}\right]$. The witness generation algorithm returns a witness $w_{\boldsymbol{B}}$ to the fact that $\boldsymbol{B}$ has been accumulated in $\operatorname{Acc}_{X}$. It first computes the matrix

$$
\boldsymbol{F}_{\boldsymbol{B}}=\left[\boldsymbol{A} \| \sum_{1 \leq i(\neq j) \leq Q^{\prime}} \boldsymbol{B}_{i}\right] \in \mathbb{Z}_{q}^{n \times\left(m+m^{\prime}\right)} .
$$

It then samples a vector $\boldsymbol{d}_{\boldsymbol{B}} \in \Lambda^{\perp}\left(\boldsymbol{F}_{\boldsymbol{B}}\right) \subseteq \mathbb{Z}^{\left(m+m^{\prime}\right)}$ following the distribution $D_{\Lambda^{\perp}\left(\boldsymbol{F}_{\boldsymbol{B}}\right), s, \mathbf{0}}$. This is done, using sk $=\boldsymbol{T}_{\boldsymbol{A}}$, as follows:

$$
\boldsymbol{d}_{\boldsymbol{B}} \leftarrow \text { SampleD }\left(\text { BasisDel }\left(\boldsymbol{T}_{\boldsymbol{A}}, \boldsymbol{A}, \sum_{1 \leq i(\neq j) \leq Q^{\prime}} \boldsymbol{B}_{i}\right), s, \mathbf{0}\right) .
$$

The witness $w_{\boldsymbol{B}}$ is set to $w_{\boldsymbol{B}}=\boldsymbol{d}_{\boldsymbol{B}}$. See Theorem 2 for a description of SampleD, and $\S$ 2.1.4 for BasisDel.

- $\operatorname{Verify}\left(\boldsymbol{B}, w_{\boldsymbol{B}}, \operatorname{Acc}_{X}\right)$ : For an element $\boldsymbol{B} \in \mathcal{M}$ the verification algorithm proceeds as follows:

- Compute

$$
\boldsymbol{F}_{\boldsymbol{B}}=\left[\boldsymbol{A} \|\left(\operatorname{Acc}_{X}-\boldsymbol{B}\right)\right] \in \mathbb{Z}_{q}^{n \times\left(m+m^{\prime}\right)}
$$

and check if $\boldsymbol{F}_{\boldsymbol{B}} \cdot w_{\boldsymbol{B}}=\mathbf{0} \bmod q$, i.e., if $w_{\boldsymbol{B}} \in \Lambda^{\perp}\left(\boldsymbol{F}_{\boldsymbol{B}}\right)$.

- Finally, check if $w_{\boldsymbol{B}}$ is small by verifying that $0<\left\|w_{\boldsymbol{B}}\right\| \leq s \sqrt{m+m^{\prime}}$.

If all the checks pass, output 1 ; otherwise, output 0 .

\subsection{Correctness}

It is easy to see by inspection that the accumulator scheme is correct, i.e., the correctly accumulated values have verifying witnesses with overwhelming probability. But for completeness we discuss the correctness of our scheme in detail.

Let $X=\left\{\boldsymbol{B}_{1}, \ldots, \boldsymbol{B}_{Q^{\prime}}\right\} \subseteq \mathcal{M}$, with corresponding accumulation value $\operatorname{Acc}_{X}=\sum_{i=1}^{Q^{\prime}} \boldsymbol{B}_{i}$. We show that every $\boldsymbol{B} \in X$ admits a verifying witness with respect to $\operatorname{Acc}_{X}$. Without loss of generality, let $\boldsymbol{B}=\boldsymbol{B}_{1}$. A valid witness for $\boldsymbol{B}_{1}$ is a short vector $\boldsymbol{d}_{\boldsymbol{B}_{1}}$ in the lattice $\Lambda^{\perp}\left(\boldsymbol{F}_{\boldsymbol{B}_{1}}\right)$ (where $\left.\boldsymbol{F}_{\boldsymbol{B}_{1}}=\left[\boldsymbol{A} \| \sum_{i=2}^{Q^{\prime}} \boldsymbol{B}_{i}\right] \in \mathbb{Z}_{q}^{n \times\left(m+m^{\prime}\right)}\right)$, i.e., $\left\|\boldsymbol{d}_{\boldsymbol{B}_{1}}\right\| \leq s \sqrt{\left(m+m^{\prime}\right)}$. Lemma 1 says that a sample in $\Lambda^{\perp}\left(\boldsymbol{F}_{\boldsymbol{B}_{1}}\right)$, following $D_{\Lambda^{\perp}\left(\boldsymbol{F}_{\left.\boldsymbol{B}_{1}\right), s, \mathbf{0}}\right.}$, has norm bounded by $s \sqrt{\left(m+m^{\prime}\right)}$ if $s \geq \omega\left(\sqrt{\log \left(m+m^{\prime}\right)}\right)$. The algorithm of Theorem 2 provides a method to sample from $D_{\Lambda^{\perp}\left(\boldsymbol{F}_{\left.\boldsymbol{B}_{1}\right), s, \mathbf{0}}\right.}$ if it is provided with a basis $\boldsymbol{T}_{\boldsymbol{F}_{\boldsymbol{B}_{1}}}$ of $\Lambda^{\perp}\left(\boldsymbol{F}_{\boldsymbol{B}_{1}}\right)$, such that $s \geq\left\|\tilde{\boldsymbol{T}}_{\boldsymbol{F}_{\boldsymbol{B}_{1}}}\right\| \cdot \omega\left(\sqrt{\log \left(m+m^{\prime}\right)}\right)$. We now see that this is indeed the case.

The witness generation algorithm has access to a short basis $\boldsymbol{T}_{\boldsymbol{A}}$ of the lattice $\Lambda^{\perp}(\boldsymbol{A})$. With $\left(\boldsymbol{T}_{\boldsymbol{A}}, \boldsymbol{A}, \sum_{i=2}^{Q^{\prime}} \boldsymbol{B}_{i}\right)$ as input, the basis delegation algorithm BasisDel of $\S 2.1 .4$ constructs a basis $\boldsymbol{T}_{\boldsymbol{F}_{B_{1}}}$ of $\Lambda^{\perp}\left(\boldsymbol{F}_{\boldsymbol{B}_{1}}\right)$ such that $\left\|\tilde{\boldsymbol{T}}_{\boldsymbol{F}_{B_{1}}}\right\|=\left\|\tilde{\boldsymbol{T}}_{\boldsymbol{A}}\right\|$. But $\left\|\tilde{\boldsymbol{T}}_{\boldsymbol{A}}\right\| \leq L \leq \frac{s}{\omega\left(\sqrt{\log \left(m+m^{\prime}\right)}\right)}$, and therefore we have $s \geq\left\|\tilde{\boldsymbol{T}}_{\boldsymbol{F}_{B_{1}}}\right\| \cdot \omega\left(\sqrt{\log \left(m+m^{\prime}\right)}\right)$.

Hence, the sampled vector $\boldsymbol{d}_{\boldsymbol{B}_{1}} \leftarrow$ SampleD $\left(\right.$ BasisDel $\left.\left(\boldsymbol{T}_{\boldsymbol{A}}, \boldsymbol{A}, \sum_{i=2}^{Q^{\prime}} \boldsymbol{B}_{i}\right), s, \mathbf{0}\right)$ constitute a valid witness for the membership of $\boldsymbol{B}_{1}$ in $X$ with respect to $\operatorname{Acc}_{X}$. 


\subsection{Security}

In the following theorem we now reduce the SIS problem to break the security of our accumulator scheme.

Theorem 3 For parameters $\lambda, n, q, m, m^{\prime}, L, s$, and $Q$, as listed in the scheme, if there is a PPT adversary $\mathcal{A}$ that breaks the one-way security of our accumulator scheme, with probability $\epsilon$, then there is a PPT algorithm $\mathcal{B}$ that solves the $\mathrm{SIS}_{q, m, \beta}$ problem with probability $\epsilon^{\prime} \geq \epsilon / 3$, for some polynomial function $\beta=\operatorname{poly}(\lambda)$; in particular $\beta=Q s^{\prime} s\left(m+m^{\prime}\right)$, where $s^{\prime} \geq \omega(\sqrt{\log m}){ }^{3}$.

Proof: Suppose that there exists such a forger $\mathcal{A}$. We construct a solver $\mathcal{B}$ that simulates an attack environment and uses an invalid element-witness pair ( $\mathcal{A}$ 's output) to create its solution for SIS problem. The various operations performed by $\mathcal{B}$ are the following.

\section{- Invocation}

$-\mathcal{B}$ is invoked on a random instance $\left(q, \boldsymbol{A} \in \mathbb{Z}_{q}^{n \times m}, \beta\right)$ of SIS problem and asked to submit a solution.

\section{- Simulation}

$-\mathcal{B}$ sets the public key pk of the accumulator scheme to pk $=\boldsymbol{A}$.

- It then picks $Q$ short random matrices $\boldsymbol{R}_{1}, \ldots, \boldsymbol{R}_{Q} \in \mathbb{Z}^{m \times m^{\prime}}$ such that $\left\|\boldsymbol{R}_{i}\right\| \leq s^{\prime} \sqrt{m}$, for some $s^{\prime} \geq \omega(\sqrt{\log m})$. It can do so, by independently sampling the columns of $\boldsymbol{R}_{i}$ 's from $D_{\mathbb{Z}^{m}, s^{\prime}, \mathbf{0}}$.

- It then sets the message space $\mathcal{M}$ to $\left\{\boldsymbol{B}_{1}=\boldsymbol{A} \boldsymbol{R}_{1} \bmod q, \ldots, \boldsymbol{B}_{Q}=\boldsymbol{A} \boldsymbol{R}_{Q} \bmod q \in\right.$ $\left.\mathbb{Z}_{q}^{n \times m^{\prime}}\right\}$. By Lemma 2 the distribution of $\boldsymbol{B}_{i}=\boldsymbol{A} \boldsymbol{R}_{i}$ is statistically close to uniform over $\mathbb{Z}_{q}^{n \times m}$ when columns of $\boldsymbol{R}_{i}$ 's are chosen from $D_{\mathbb{Z}^{m}, s^{\prime}, \mathbf{0}}$.

- Finally, $\mathcal{B}$ gives $(\boldsymbol{A}, \mathcal{M})$ to $\mathcal{A}$.

\section{- Breaking One-way Security}

- $\mathcal{A}$ outputs $\left(X^{*}=\left\{\boldsymbol{B}_{i_{1}}, \ldots, \boldsymbol{B}_{i_{k}}\right\} \subseteq \mathcal{M}, \boldsymbol{B}^{*}=\boldsymbol{B}_{\ell} \in \mathcal{M}, w_{\boldsymbol{B}}^{*} \in \mathbb{Z}^{m+m^{\prime}}\right)$ such that

$$
\boldsymbol{B}^{*} \notin X^{*} \text { and Verify }\left(\boldsymbol{B}^{*}, w_{\boldsymbol{B}}^{*}, \operatorname{Acc}_{X^{*}} \leftarrow \operatorname{Acc}\left(X^{*}\right)\right)=1 \text {. }
$$

\section{- Solving SIS Instance}

- $\operatorname{Verify}\left(\boldsymbol{B}^{*}, w_{\boldsymbol{B}}^{*}, \operatorname{Acc}_{X^{*}}\right)=1$ means

$$
w_{\boldsymbol{B}}^{*} \in \Lambda^{\perp}\left(\boldsymbol{A} \|\left(\operatorname{Acc}_{X^{*}}-\boldsymbol{B}^{*}\right)\right), \text { and } 0<\left\|w_{\boldsymbol{B}}^{*}\right\| \leq s \sqrt{m+m^{\prime}}
$$

- Compute $\boldsymbol{R}^{*}=\sum_{j=1}^{k} \boldsymbol{R}_{i_{j}}-\boldsymbol{R}_{\ell}$. Also, write $w_{\boldsymbol{B}}^{*} \in \mathbb{Z}^{m+m^{\prime}}$ as $\left[\begin{array}{c}w_{\boldsymbol{B}}^{* \prime} \\ w_{\boldsymbol{B}}^{* \prime \prime}\end{array}\right]$ such that $w_{\boldsymbol{B}}^{* \prime} \in \mathbb{Z}^{m}, w_{\boldsymbol{B}}^{* \prime \prime} \in \mathbb{Z}^{m^{\prime}}$.

- Finally, $\mathcal{B}$ outputs $\boldsymbol{e}=w_{\boldsymbol{B}}^{* \prime}+\boldsymbol{R}^{*} w_{\boldsymbol{B}}^{* \prime \prime} \in \mathbb{Z}^{m}$ as solution to SIS instance $\left(q, \boldsymbol{A} \in \mathbb{Z}_{q}^{n \times m}, \beta\right)$.

We now show that $\boldsymbol{e}$ is indeed a valid solution (with probability greater than $2 / 3$ ), i.e., $\boldsymbol{A} \boldsymbol{e}=$ $\mathbf{0} \bmod q,\|\boldsymbol{e}\| \leq \beta$, and $\boldsymbol{e} \neq \mathbf{0}$. Clearly $\boldsymbol{A} \boldsymbol{e}=\boldsymbol{A}\left(w_{\boldsymbol{B}}^{* \prime}+\boldsymbol{R}^{*} w_{\boldsymbol{B}}^{* \prime \prime}\right)=\boldsymbol{A} w_{\boldsymbol{B}}^{* \prime}+\left(\sum_{j=1}^{k} \boldsymbol{A} \boldsymbol{R}_{i_{j}}\right.$ $\left.\boldsymbol{A R}_{\ell}\right) w_{\boldsymbol{B}}^{* \prime \prime}=\boldsymbol{A} w_{\boldsymbol{B}}^{* \prime}+\left(\sum_{j=1}^{k} \boldsymbol{B}_{i_{j}}-\boldsymbol{B}_{\ell}\right) w_{\boldsymbol{B}}^{* \prime \prime}=\left[\boldsymbol{A} \|\left(\mathrm{Acc}_{X^{*}}-\boldsymbol{B}^{*}\right)\right] w_{\boldsymbol{B}}^{*}=\mathbf{0} \bmod q$.

\footnotetext{
${ }^{3}$ To ensure that the SIS instance with norm bound $\beta=Q s^{\prime} s\left(m+m^{\prime}\right)$ is hard (worst-case to average-case reduction), the modulus $q$ of the scheme should satisfy $q>\beta \cdot w(\sqrt{n \log n}$ ) (See Theorem 1). In particular, for $q$ we choose the smallest prime bigger than $\lambda^{t}$ for the smallest $t$ such that $q>\beta \cdot \omega(\sqrt{n \log n})$. Choosing $n \log n$ for $\omega(\sqrt{n \log n})$, implies $\beta \cdot \omega(\sqrt{n \log n})=\operatorname{poly}(\lambda)$, as $Q, s^{\prime}, s, m, m^{\prime}, n$ are all bounded above by a poly $(\lambda)$ size number.
} 
Next, we show that $\|\boldsymbol{e}\| \leq \beta$. We have $\boldsymbol{e}=w_{\boldsymbol{B}}^{* \prime}+\boldsymbol{R}^{*} w_{\boldsymbol{B}}^{* \prime \prime}$, where $\boldsymbol{R}^{*}=\sum_{j=1}^{k} \boldsymbol{R}_{i_{j}}-\boldsymbol{R}_{\ell}$ is a sum of $k$ low norm matrices $\boldsymbol{R}_{i_{j}}$ minus a low norm matrix $\boldsymbol{R}_{\ell}\left(\left\|\boldsymbol{R}_{i}\right\| \leq s^{\prime} \sqrt{m}\right.$ with overwhelming probability). Therefore we have,

$$
\begin{aligned}
\|\boldsymbol{e}\| & =\left\|w_{\boldsymbol{B}}^{* \prime}+\left(\sum_{j=1}^{k} \boldsymbol{R}_{i_{j}}-\boldsymbol{R}_{\ell}\right) w_{\boldsymbol{B}}^{* \prime \prime}\right\| \\
& \leq\left\|w_{\boldsymbol{B}}^{* \prime}\right\|+\left\|\sum_{j=1}^{k} \boldsymbol{R}_{i_{j}}-\boldsymbol{R}_{\ell}\right\|\left\|w_{\boldsymbol{B}}^{* \prime \prime}\right\| \\
& \leq s \sqrt{m+m^{\prime}}\left(1+(k+1) s^{\prime} \sqrt{m}\right) \\
& \leq Q s^{\prime} s\left(m+m^{\prime}\right) .
\end{aligned}
$$

We now complete the proof by showing that $\boldsymbol{e}=w_{\boldsymbol{B}}^{* \prime}+\boldsymbol{R}^{*} w_{\boldsymbol{B}}^{* \prime \prime} \neq \mathbf{0}$. Let us assume $w_{\boldsymbol{B}}^{* \prime \prime} \neq \mathbf{0}$ (as $w_{\boldsymbol{B}}^{*} \neq \mathbf{0}, w_{\boldsymbol{B}}^{* \prime \prime}=\mathbf{0}$ implies $w_{\boldsymbol{B}}^{* \prime} \neq \mathbf{0}$ and thus $\boldsymbol{e} \neq \mathbf{0}$ ). As $0<\left\|w_{\boldsymbol{B}}^{* \prime \prime}\right\| \leq s \sqrt{m+m^{\prime}}<<q$, there must be at least one coordinate of $w_{B}^{* \prime \prime}$ that is non-zero modulo $q$. W.l.o.g., let this coordinate be the first one in $w_{\boldsymbol{B}}^{* \prime \prime}$, and call it $z$. Let $\boldsymbol{r}_{1}^{*}$ be the first column of $\boldsymbol{R}^{*}$, and let $\boldsymbol{r}_{t 1}$ be the first column of $\boldsymbol{R}_{t}$ for each $t$ in $\left\{i_{1}, \ldots, i_{k}, \ell\right\}$. Clearly, $\boldsymbol{r}_{1}^{*}=\sum_{j=1}^{k} \boldsymbol{r}_{i_{j} 1}-\boldsymbol{r}_{\ell 1}$. We focus on $\boldsymbol{r}_{i_{1} 1}$. Let $\boldsymbol{u}=z \boldsymbol{r}_{i_{1} 1}$. Rewrite $\boldsymbol{e}$ as $\boldsymbol{e}=z \boldsymbol{r}_{1}^{*}+\boldsymbol{e}^{\prime}=\boldsymbol{u}+\boldsymbol{e}^{\prime \prime}$ such that $\boldsymbol{u}$ depends on $\boldsymbol{r}_{i_{1} 1}$ and $\boldsymbol{e}^{\prime \prime}$ does not. Now, the only information about $\boldsymbol{r}_{i_{1} 1}$ available to $\mathcal{A}$ is contained in the first column of $\boldsymbol{B}_{i_{1}}=\boldsymbol{A} \boldsymbol{R}_{i_{1}}$. With even $\boldsymbol{A}$ being known in the worst case, by a simple pigeonhole principle, there are a very large (exponential in $m-n$ ) number of admissible and equally likely vectors $\boldsymbol{r}_{i_{1} 1}$, in particular more than $3 Q$ of them, that are compatible with the view of $\mathcal{A}$. At most one such value can result in cancellation of $\boldsymbol{e}$, for if some $\boldsymbol{u}$ caused all coordination of $\boldsymbol{e}$ to cancel, then every other $\boldsymbol{u}$ would fail to do so. Therefore $\operatorname{Pr}[\boldsymbol{e}=\mathbf{0}] \leq 1 / 3 Q$. Since $\mathcal{A}$ can choose $\boldsymbol{B}_{i_{1}}$ among $Q$ possible values $(Q=|\mathcal{M}|)$, it follows that $\mathcal{A}$ can know the value of $\boldsymbol{u}$ with probability at most $1 / 3$. Hence, $\operatorname{Pr}[\boldsymbol{e} \neq \mathbf{0}] \geq 2 / 3$.

Therefore, if $\mathcal{A}$ breaks the one-way security of the scheme with probability $\epsilon$, then $\mathcal{B}$ solves the SIS instance with probability $\epsilon^{\prime} \geq 2 \epsilon / 3$.

\section{Conclusion and Open Problems}

We have provided the first lattice-based construction of a one-way accumulator scheme and proved its security from hardness assumption of the SIS problem (which is itself implied by worst-case lattice assumption). We leave open the problem of how to extend our basic scheme in order to incorporate dynamic and universal functionalities. Another interesting problem is to extend our scheme such that zero-knowledge proofs of membership can be obtained.

\section{References}

[ABB10] Shweta Agrawal, Dan Boneh, and Xavier Boyen. Efficient lattice (H)IBE in the standard model. In EUROCRYPT, volume 6110 of Lecture Notes in Computer Science, pages 553-572. Springer, 2010. Full Version at "http://crypto.stanford. edu/ dabo/pubs/papers/latticebb.pdf'.

$\left[\mathrm{ABC}^{+} 12\right]$ Jae Hyun Ahn, Dan Boneh, Jan Camenisch, Susan Hohenberger, Abhi Shelat, and Brent Waters. Computing on authenticated data. In TCC, volume 7194 of Lecture Notes in Computer Science, pages 1-20. Springer, 2012. 
[Ajt96] Miklós Ajtai. Generating hard instances of lattice problems (extended abstract). In STOC, pages 99-108, 1996.

[Ajt99] Miklós Ajtai. Generating hard instances of the short basis problem. In ICALP, volume 1644 of Lecture Notes in Computer Science, pages 1-9. Springer, 1999.

[AP09] Joël Alwen and Chris Peikert. Generating shorter bases for hard random lattices. In STACS, volume 3 of LIPIcs. Schloss Dagstuhl - Leibniz-Zentrum fuer Informatik, Germany, 2009.

[BdM93] Josh Cohen Benaloh and Michael de Mare. One-way accumulators: A decentralized alternative to digital sinatures (extended abstract). In EUROCRYPT, volume 765 of Lecture Notes in Computer Science, pages 274-285. Springer, 1993.

[BLL00] Ahto Buldas, Peeter Laud, and Helger Lipmaa. Accountable certificate management using undeniable attestations. In $A C M C C S$, pages 9-17, 2000.

[Boy10] Xavier Boyen. Lattice mixing and vanishing trapdoors: A framework for fully secure short signatures and more. In PKC, volume 6056 of Lecture Notes in Computer Science, pages 499-517. Springer, 2010.

[BP97] Niko Bari and Birgit Pfitzmann. Collision-free accumulators and fail-stop signature schemes without trees. In EUROCRYPT, volume 1233 of Lecture Notes in Computer Science, pages 480-494. Springer, 1997.

[CF13] Dario Catalano and Dario Fiore. Vector commitments and their applications. In PKC, volume 7778 of Lecture Notes in Computer Science, pages 55-72. Springer, 2013.

[CHKO12] Philippe Camacho, Alejandro Hevia, Marcos Kiwi, and Roberto Opazo. Strong accumulators from collision-resistant hashing. International Journal of Information Security, 11(5):349-363, 2012.

[CHKP12] David Cash, Dennis Hofheinz, Eike Kiltz, and Chris Peikert. Bonsai trees, or how to delegate a lattice basis. J. Cryptology, 25(4):601-639, 2012.

[CJ10] Sébastien Canard and Amandine Jambert. On extended sanitizable signature schemes. In CT-RSA, volume 5985 of Lecture Notes in Computer Science, pages 179-194. Springer, 2010.

[CKS09] Jan Camenisch, Markulf Kohlweiss, and Claudio Soriente. An accumulator based on bilinear maps and efficient revocation for anonymous credentials. In $P K C$, volume 5443 of Lecture Notes in Computer Science, pages 481-500. Springer, 2009.

[CL02] Jan Camenisch and Anna Lysyanskaya. Dynamic accumulators and application to efficient revocation of anonymous credentials. In CRYPTO, volume 2442 of Lecture Notes in Computer Science, pages 61-76. Springer, 2002.

[DKNS04] Yevgeniy Dodis, Aggelos Kiayias, Antonio Nicolosi, and Victor Shoup. Anonymous identification in ad hoc groups. In EUROCRYPT, volume 3027 of Lecture Notes in Computer Science, pages 609-626. Springer, 2004.

[DT08] Ivan Damgård and Nikos Triandopoulos. Supporting non-membership proofs with bilinear-map accumulators. IACR Cryptology ePrint Archive, 2008:538, 2008. 
[Gen09] Craig Gentry. Fully homomorphic encryption using ideal lattices. In STOC, pages 169-178. ACM, 2009.

[GPV08] Craig Gentry, Chris Peikert, and Vinod Vaikuntanathan. Trapdoors for hard lattices and new cryptographic constructions. In Cynthia Dwork, editor, STOC 2008, pages 197-206. ACM, 2008.

[LLX] Jiangtao Li, Ninghui Li, and Rui Xue. Universal accumulators with efficient nonmembership proofs. In $A C N S$, Lecture Notes in Computer Science.

[LM06] Vadim Lyubashevsky and Daniele Micciancio. Generalized compact knapsacks are collision resistant. In ICALP, volume 4052 of Lecture Notes in Computer Science, pages 144-155. Springer, 2006.

[MGGR] Ian Miers, Christina Garman, Matthew Green, and Aviel D. Rubin. Zerocoin: Anonymous distributed e-cash from bitcoin. In IEEE Symposium on Security and Privacy. IEEE Computer Society.

[MP12] Daniele Micciancio and Chris Peikert. Trapdoors for lattices: Simpler, tighter, faster, smaller. In EUROCRYPT, volume 7237 of Lecture Notes in Computer Science, pages 700-718. Springer, 2012.

[MR07] Daniele Micciancio and Oded Regev. Worst-case to average-case reductions based on gaussian measures. SIAM J. Comput., 37(1):267-302, 2007.

[Ngu05] Lan Nguyen. Accumulators from bilinear pairings and applications. In CT-RSA, volume 3376 of Lecture Notes in Computer Science, pages 275-292. Springer, 2005.

[Nyb96] Kaisa Nyberg. Fast accumulated hashing. In FSE, volume 1039 of Lecture Notes in Computer Science, pages 83-87. Springer, 1996.

[PR06] Chris Peikert and Alon Rosen. Efficient collision-resistant hashing from worst-case assumptions on cyclic lattices. In TCC, volume 3876 of Lecture Notes in Computer Science, pages 145-166. Springer, 2006.

[PS14] Henrich Christopher Pöhls and Kai Samelin. On updatable redactable signatures. In ACNS, volume 8479 of Lecture Notes in Computer Science, pages 457-475. Springer, 2014.

[PV08] Chris Peikert and Vinod Vaikuntanathan. Noninteractive statistical zero-knowledge proofs for lattice problems. In CRYPTO, volume 5157 of Lecture Notes in Computer Science, pages 536-553. Springer, 2008.

[WWP08] Peishun Wang, Huaxiong Wang, and Josef Pieprzyk. Improvement of a dynamic accumulator at ICICS 07 and its application in multi-user keyword-based retrieval on encrypted data. In $A P S C C$, pages 1381-1386. IEEE Computer Society, 2008. 